\title{
A Non-standard Version of the Borsuk-Ulam Theorem
}

\author{
by
}

\author{
Carlos BIASI and Denise de MATTOS
}

Presented by Czestaw BESSAGA

Summary. E. Pannwitz showed in 1952 that for any $n \geq 2$, there exist continuous maps $\varphi: S^{n} \rightarrow S^{n}$ and $f: S^{n} \rightarrow \mathbb{R}^{2}$ such that $f(x) \neq f(\varphi(x))$ for any $x \in S^{n}$. We prove that, under certain conditions, given continuous maps $\psi, \varphi: X \rightarrow X$ and $f: X \rightarrow \mathbb{R}^{2}$, although the existence of a point $x \in X$ such that $f(\psi(x))=f(\varphi(x))$ cannot always be assured, it is possible to establish an interesting relation between the points $f(\varphi \psi(x)), f\left(\varphi^{2}(x)\right)$ and $f\left(\psi^{2}(x)\right)$ when $f(\varphi(x)) \neq f(\psi(x))$ for any $x \in X$, and a non-standard version of the Borsuk-Ulam theorem is obtained.

1. Introduction. Let $X$ be a topological space. An involution on $X$ is a continuous map $\varphi: X \rightarrow X$ which is its own inverse. A classical example is the antipodal map $A: S^{n} \rightarrow S^{n}, A(x)=-x$, where $S^{n}$ denotes the $n$-dimensional sphere; the points $x$ and $A(x)$ are said to be antipodal points. The classical Borsuk-Ulam theorem [1] states that every continuous map $f$ from $S^{n}$ into $\mathbb{R}^{n}$ collapses at least a pair of antipodal points, that is, there exists a point $x \in S^{n}$ such that $f(x)=f(A(x))$.

Several generalizations of this theorem, in various directions, are well known. In some of these generalizations the sphere is replaced by a more general space $X$ and the antipodal map is replaced by an involution $T: X \rightarrow X$ which is free, that is, $T(x) \neq x$ for any $x \in X$. In this direction see, for example, the references $[2,8,9]$.

Let us now replace the domain $S^{n}$ by a topological space $X$ and the identity and the antipodal map on $S^{n}$ by a pair of any continuous maps $\psi, \varphi$ on $X$. A question that naturally arises is whether or not for every continuous map $f: X \rightarrow \mathbb{R}^{n}$ there exists a point $x \in X$ such that $f(\psi(x))=f(\varphi(x))$.

2000 Mathematics Subject Classification: Primary 54H25; Secondary 55M20, 47H09.

Key words and phrases: Borsuk-Ulam theorem, involution, $\alpha$-contraction.

The second author was supported by FAPESP of Brasil Grant 01/02226-9. 
We first consider the one-dimensional case. If $X$ is a compact and connected space, then for every continuous map $f: X \rightarrow \mathbb{R}$ it is possible to show that there exists a point $x \in X$ such that

$$
f(\psi(x))=f(\varphi(x)) .
$$

The proof is elementary. However, for $n=2, X=S^{k}$ and $\psi=\operatorname{Id}_{S^{k}}$ the answer is negative. E. Pannwitz proved in [7] that for any $k \geq 2$, there exist continuous maps $\varphi: S^{k} \rightarrow S^{k}$ and $f: S^{k} \rightarrow \mathbb{R}^{2}$ such that $f(x) \neq f(\varphi(x))$ for any $x \in S^{k}$.

In this paper, our objective is to show that, under certain conditions, for a given continuous map $f: X \rightarrow \mathbb{R}^{2}$, although the existence of a point $x \in X$ such that (1.1) holds cannot always be assured, it is possible to establish an interesting relation between the points

$$
u=f(\psi \varphi(x)), \quad v=f\left(\varphi^{2}(x)\right), \quad w=f\left(\psi^{2}(x)\right)
$$

when $f(\varphi(x)) \neq f(\psi(x))$ for any $x \in X$. In general, such points are vertices of a triangle in $\mathbb{R}^{2}$ and we prove that this triangle degenerates to a closed line segment determined by the vertices $v$ and $w$ for, at least, a point $x$ in a special subset of $X$. The existence of such a subset is ensured when $X$ is a complete metric space and $\varphi$ is an $\alpha$-contraction on $X$, where $\alpha$ is the measure of noncompactness.

When $\psi$ is the identity map and $\varphi$ is a free involution on $X$, we obtain a version of the Borsuk-Ulam theorem in the two-dimensional case.

We denote by $[v, w]$ the closed line segment in $\mathbb{R}^{2}$ joining the points $v$ and $w$. We will specifically prove the following

THEOREM 1.1. Let $X$ be a Hausdorff space and $A$ a compact, connected and locally pathwise connected subset of $X$. Let $\psi, \varphi: X \rightarrow X$ be continuous maps such that $A$ is invariant under $\psi$ and $\varphi$, that is, $\psi(A) \subset A$ and $\varphi(A) \subset A$. Suppose that

(i) $\psi_{*}-\varphi_{*}: i_{*}\left(H_{1}(A, \mathbb{Q})\right) \rightarrow i_{*}\left(H_{1}(A, \mathbb{Q})\right)$ is a surjective map;

(ii) $(\psi \circ \varphi)(x)=(\varphi \circ \psi)(x)$ for any $x \in A$.

Then for every continuous map $f: X \rightarrow \mathbb{R}^{2}$, either there exists a point $x \in X$ such that $f(\varphi(x))=f(\psi(x))$ or there exists a point $x \in A$ such that $f(\varphi \psi(x)) \in\left[f\left(\varphi^{2}(x)\right), f\left(\psi^{2}(x)\right)\right]$.

2. Proof of Theorem 1.1. For the proof of Theorem 1.1, we need the following

Lemma 2.1. Let $X$ be a connected space and $K \neq \emptyset$ a compact subset of $X$. Let $g_{1}, g_{2}: X \rightarrow \mathbb{R}$ be continuous maps such that $g_{1}(K) \subset g_{2}(K)$. Then there exists a point $x \in X$ such that $g_{1}(x)=g_{2}(x)$. 
Proof. Consider the continuous map $h: X \rightarrow \mathbb{R}$ given by $h(x)=$ $g_{2}(x)-g_{1}(x)$ for any $x \in X$. Since $K$ is compact, there exist $x_{0}, x_{1} \in K$ such that

$$
g_{2}\left(x_{0}\right) \leq g_{2}(x) \leq g_{2}\left(x_{1}\right)
$$

for any $x \in K$. Furthermore, $g_{1}(x) \in g_{2}(K)$ for any $x \in K$ and it follows from (2.1) that

$$
g_{2}\left(x_{0}\right) \leq g_{1}(x) \leq g_{2}\left(x_{1}\right), \quad \forall x \in K,
$$

which implies that $h\left(x_{0}\right) \leq 0 \leq h\left(x_{1}\right)$ and consequently there is an $x \in X$ such that $h(x)=0$, that is, $g_{1}(x)=g_{2}(x)$.

As a direct consequence we obtain the following

Corollary 2.2. Let $X$ be a connected space and $K$ a compact subset of $X$. Let $\psi, \varphi: X \rightarrow X$ be continuous maps such that $\psi(K) \subset \varphi(K)$. Then for every continuous map $g: X \rightarrow \mathbb{R}$ there exists a point $x \in X$ such that $g(\psi(x))=g(\varphi(x))$.

Lemma 2.3. Let $X$ be a topological space and let $f, g: X \rightarrow S^{n}$ be continuous maps. Suppose that there exists $u \in H_{n}(X, \mathbb{Z})$ such that $f_{*}(u) \neq$ $(-1)^{n+1} g_{*}(u)$. Then there exists $x \in X$ such that $f(x)=g(x)$.

Proof. Suppose that $f(x) \neq g(x)$ for any $x \in X$. Then the line segment in $\mathbb{R}^{n+1}$ from $f(x)$ to $-g(x)$ does not pass through the origin, since otherwise these points would be antipodal and consequently $f(x)=g(x)$. Hence we can define a map $F: X \times I \rightarrow S^{n}$ by

$$
F(x, t)=\frac{(1-t)(-g(x))+t \cdot f(x)}{\|(1-t)(-g(x))+t \cdot f(x)\|}, \quad \forall(x, t) \in X \times I,
$$

which is a homotopy between $f$ and $-g=A \circ g$, where $A: S^{n} \rightarrow S^{n}$ denotes the antipodal map, whose degree is $(-1)^{n+1}$. It follows that for any $u \in H^{n}(X, \mathbb{Z}), f_{*}(u)=(-1)^{n+1} g_{*}(u)$.

Proof of Theorem 1.1. Suppose that $f(\varphi(x)) \neq f(\psi(x))$ for any $x \in X$. Then we can define a continuous map $h: X \rightarrow S^{1}$ by

$$
h(x)=\frac{f(\psi(x))-f(\varphi(x))}{\|f(\psi(x))-f(\varphi(x))\|} .
$$

Let $g: A \rightarrow S^{1}$ be the restriction of $h$ to $A$. It suffices to show the existence of a point $x \in A$ such that $g(\varphi(x))=g(\psi(x))$ or equivalently,

$$
\frac{f(\psi \varphi(x))-f\left(\varphi^{2}(x)\right)}{\left\|f(\psi \varphi(x))-f\left(\varphi^{2}(x)\right)\right\|}=\frac{f\left(\psi^{2}(x)\right)-f(\varphi \psi(x))}{\left\|f\left(\psi^{2}(x)\right)-f(\varphi \psi(x))\right\|} .
$$

In fact, for any $x \in A$ set $u=f(\psi \varphi(x))=f(\varphi \psi(x)), v=f\left(\varphi^{2}(x)\right)$ and 
$w=f\left(\psi^{2}(x)\right)$. Then $(2.3)$ is equivalent to

and so

$$
\frac{u-v}{\|u-v\|}=\frac{w-u}{\|w-u\|}
$$

$$
u=\left(\frac{\|u-v\|}{\|u-v\|+\|w-u\|}\right) w+\left(\frac{\|w-u\|}{\|u-v\|+\|w-u\|}\right) v,
$$

that is, $u=f(\psi \varphi(x))$ belongs to the line segment in $\mathbb{R}^{2}$ from $v=f\left(\varphi^{2}(x)\right)$ to $w=f\left(\psi^{2}(x)\right)$.

Let $h_{*}: H_{1}(X, \mathbb{Q}) \rightarrow H_{1}\left(S^{1}, \mathbb{Q}\right)$. There are two cases to consider:

$(1)$ there exists $v \in i_{*}\left(H_{1}(A, \mathbb{Q})\right)$ such that $h_{*}(v) \neq 0$,

(2) $h_{*}(v)=0$ for any $v \in i_{*}\left(H_{1}(A, \mathbb{Q})\right)$.

In the first case, since $\psi_{*}-\varphi_{*}$ is surjective, there exists $u \in i_{*}\left(H_{1}(A, \mathbb{Q})\right)$ such that $v=\psi_{*}(u)-\varphi_{*}(u)$. Then

$h_{*}(v)=h_{*}\left(\psi_{*}(u)-\varphi_{*}(u)\right)=g_{*}\left(\psi_{*}(u)-\varphi_{*}(u)\right)=(g \circ \psi)_{*}(u)-(g \circ \varphi)_{*}(u) \neq 0$, which implies that $(g \circ \psi)_{*}(u) \neq(g \circ \varphi)_{*}(u)$. It follows from Lemma 2.3 that there exists $x \in A$ such that $g(\psi(x))=g(\varphi(x))$.

Now suppose that $h_{*}(v)=0$ for any $v \in i_{*}\left(H_{1}(A, \mathbb{Q})\right)$ and let $u \in H_{1}(A, \mathbb{Q})$; then $i_{*}(u)=v \in i_{*}\left(H_{1}(A, \mathbb{Q})\right)$ and thus

$$
h_{*}(v)=h_{*}\left(i_{*}(u)\right)=(h \circ i)_{*}(u)=g_{*}(u)=0,
$$

that is, $g_{*}: H_{1}(A, \mathbb{Q}) \rightarrow H_{1}\left(S^{1}, \mathbb{Q}\right)$ is the zero map, which implies that $g_{*}: H_{1}(A, \mathbb{Z}) \rightarrow H_{1}\left(S^{1}, \mathbb{Z}\right)$ is also trivial.

It follows from the commutative diagram

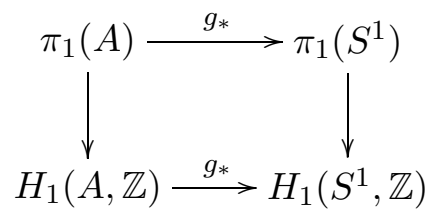

where the vertical arrows denotes the Hurewicz homomorphism, that $g_{*}: \pi_{1}(A) \rightarrow \pi_{1}\left(S^{1}\right)$ is the zero map. Since $A$ is Hausdorff and locally pathwise connected, by the lifting theorem (see, for example, [5, p. 89] and $[4$, p. 26, Theorem 6.1]) there exists $\tilde{g}: A \rightarrow \mathbb{R}$ such that the diagram

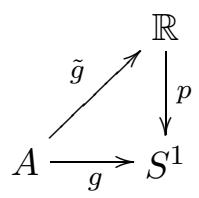

is commutative, where $p: \mathbb{R} \rightarrow S^{1}$ is the universal covering. On the other hand, since $A$ is invariant under $\varphi$, we obtain the sequence $\left\{\varphi^{n}(A)\right\}_{n \in \mathbb{N}}$ of subsets of $A$ such that 


$$
\cdots \subset \varphi^{n}(A) \subset \varphi^{n-1}(A) \subset \cdots \subset \varphi^{2}(A) \subset \varphi(A) \subset A .
$$

We consider the following compact subset of $A$ :

$$
K=\bigcap_{n \in \mathbb{N}} \varphi^{n}(A),
$$

and we observe that $\psi(K) \subset K=\varphi(K)$. In fact, by hypothesis $A$ is invariant under $\psi$ and $\varphi$. Furthermore, $\varphi \circ \psi=\psi \circ \varphi$ on $A$. Thus

$\psi(K)=\psi\left(\bigcap_{n \in \mathbb{N}} \varphi^{n}(A)\right) \subset \bigcap_{n \in \mathbb{N}} \psi\left(\varphi^{n}(A)\right) \subset \bigcap_{n \in \mathbb{N}} \varphi^{n}(\psi(A)) \subset \bigcap_{n \in \mathbb{N}} \varphi^{n}(A)=K$.

It follows from Corollary 2.2 that there exists a point $x \in A$ such that $\tilde{g}(\varphi(x))=\tilde{g}(\psi(x))$. Then $p \circ \tilde{g}(\varphi(x))=p \circ \tilde{g}(\psi(x))$, which implies that $g(\varphi(x))=g(\psi(x))$, and the result follows.

We have the following immediate corollary:

Corollary 2.4. Let $X$ be a Hausdorff space and $A$ a compact, connected and locally pathwise connected subset of $X$. Let $\varphi: X \rightarrow X$ be a free involution such that $\varphi(A) \subset A$. Suppose that $\operatorname{Id}_{*}-\varphi_{*}: i_{*}\left(H_{1}(A, \mathbb{Q})\right) \rightarrow$ $i_{*}\left(H_{1}(A, \mathbb{Q})\right)$ is a surjective map. Then for every continuous map $f: X \rightarrow \mathbb{R}^{2}$ there exists $x \in X$ such that $f(x)=f(\varphi(x))$.

Remark 2.5. When $A=X=S^{2}$ and $\varphi$ is the antipodal map, we obtain the classical Borsuk-Ulam theorem in the two-dimensional case.

We observe that when $i_{*}\left(H_{1}(A, \mathbb{Q})\right)$ is the trivial group, the homomorphism $\psi_{*}-\varphi_{*}$ must be surjective. Example 2.6 illustrates this case.

EXAmPLE 2.6. Let $T_{n}=T \sharp \cdots \sharp T$ be the $n$-fold connected sum of tori, which is embedded in $\mathbb{R}^{3}$ symmetrically with respect to the origin. Let $\varphi$ : $T_{n} \rightarrow T_{n}$ be the antipodal map. If $n$ is even, there exists a loop $A$ in $T_{n}$, homologous to zero, which separates $T_{n}$ into two components symmetrical with respect to the origin such that $\varphi(A)=A$, as indicated in Figure 1.

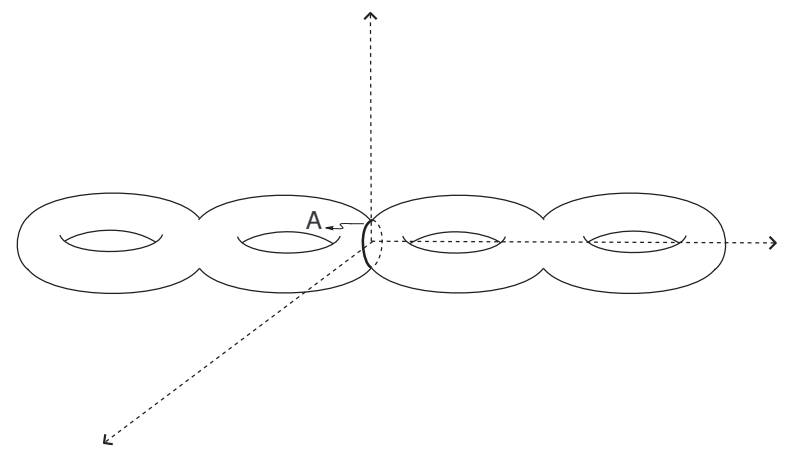

Fig. 1 
The group $i_{*}\left(H_{1}(A, \mathbb{Q})\right)$ is trivial, and so by Corollary 2.4, for every continuous map $f: T_{n} \rightarrow \mathbb{R}^{2}$ there exists a point $x \in T_{n}$ such that $f(x)=$ $f(\varphi(x))$. If $n$ is odd, one can show that this is not true.

REMARK 2.7. The referee remarked that it is possible to show the existence of a point $x \in T_{n}$ such that $f(x)=f(\varphi(x))$ by using the Yang-Smith index.

REMARK 2.8. In [8, Theorem A], we prove that if $(X, T)$ is a free involution and $X$ is pathwise connected such that $H_{r}\left(X, \mathbb{Z}_{2}\right)=0$ for $1 \leq r \leq n-1$, then for every continuous map $f: X \rightarrow \mathbb{R}^{k}$ with $k \leq n$ there exists a point $x \in X$ such that $f(x)=f(T(x))$. We observe that the above example cannot be obtained from that theorem, since $H_{1}\left(T_{n}, \mathbb{Z}_{2}\right) \neq 0$.

TheOREM 2.9. Let $X$ be a Hausdorff space and $A$ a compact, connected and locally pathwise connected subset of $X$. Let $\varphi: X \rightarrow X$ be a continuous map such that $\varphi(A) \subset A$. Suppose that $\operatorname{Id}_{*}-\varphi_{*}: i_{*}\left(H_{1}(A, \mathbb{Q})\right) \rightarrow$ $i_{*}\left(H_{1}(A, \mathbb{Q})\right)$ is a surjective map. Then for every continuous map $g: X \rightarrow \mathbb{R}$ there exists $x \in X$ such that

$$
\begin{aligned}
& g(x) \leq g(\varphi(x)) \leq g\left(\varphi^{2}(x)\right) \leq g\left(\varphi^{3}(x)\right) \quad \text { or } \\
& g(x) \geq g(\varphi(x)) \geq g\left(\varphi^{2}(x)\right) \geq g\left(\varphi^{3}(x)\right) .
\end{aligned}
$$

Proof. Consider the continuous map $f: X \rightarrow \mathbb{R}^{2}$ given by

$$
f(x)=(g(x), g(\varphi(x))), \quad \forall x \in X .
$$

By Theorem 1.1, there exists $x \in X$ such that $f(\varphi(x))$ belongs to the closed line segment in $\mathbb{R}^{2}$ from $f\left(\varphi^{2}(x)\right)$ to $f(x)$. Suppose that $f(\varphi(x))=f(x)$; this implies that

$$
g(x)=g(\varphi(x))=g\left(\varphi^{2}(x)\right) .
$$

Since $g\left(\varphi^{2}(x)\right) \leq g\left(\varphi^{3}(x)\right)$ or $g\left(\varphi^{2}(x)\right) \geq g\left(\varphi^{3}(x)\right)$, the result follows. The proof remains the same when $f(\varphi(x))=f\left(\varphi^{2}(x)\right)$.

Now, suppose that $f(\varphi(x)) \neq f(x)$ and $f(\varphi(x)) \neq f\left(\varphi^{2}(x)\right)$. Then $f(\varphi(x))$ belongs to the open line segment in $\mathbb{R}^{2}$ from $f\left(\varphi^{2}(x)\right)$ to $f(x)$, that is, there exists $0<\lambda<1$ such that $f(\varphi(x))=f(x)+\lambda\left(f\left(\varphi^{2}(x)\right)-f(x)\right)$. Thus,

$$
\begin{aligned}
g \varphi(x) & =g(x)+\lambda\left(g \varphi^{2}(x)-g(x)\right), \\
g \varphi^{2}(x) & =g \varphi(x)+\lambda\left(g \varphi^{3}(x)-g \varphi(x)\right),
\end{aligned}
$$

which implies the required alternative of inequalities.

We have the following immediate corollary:

Corollary 2.10. Let $X$ be a Hausdorff space and $A$ a compact, connected and locally pathwise connected subset of $X$. Let $\varphi: X \rightarrow X$ be a 
continuous map such that $\varphi(A) \subset A$ and $\varphi^{3}=\operatorname{Id}_{X}$. Suppose that

$$
\operatorname{Id}_{*}-\varphi_{*}: i_{*}\left(H_{1}(A, \mathbb{Q})\right) \rightarrow i_{*}\left(H_{1}(A, \mathbb{Q})\right)
$$

is a surjective map. Then for every continuous map $g: X \rightarrow \mathbb{R}$ there exists a point $x \in X$ such that $g(x)=g(\varphi(x))=g\left(\varphi^{2}(x)\right)$.

EXAMPLE 2.11. Let $S^{3}$ be the 3-dimensional standard sphere in complex 2-space $\mathbb{C}^{2}$. Let $\varphi: S^{3} \rightarrow S^{3}$ be the transformation defined by

$$
\varphi\left(z_{0}, z_{1}\right)=\left(e^{2 \pi i / 3} z_{0}, e^{2 \pi i / 3} z_{1}\right),
$$

where $z_{0}, z_{1}$ are complex numbers with $\sum_{i=0}^{1}\left|z_{i}\right|=1$. Then $\varphi$ acts freely on $S^{3}$ and generates the cyclic group $\mathbb{Z}_{3}$.

Since $H_{1}\left(S^{3}, \mathbb{Q}\right)=0$, we see that $\operatorname{Id}_{*}-\varphi_{*}$ is surjective. It follows from Corollary 2.10 that for every continuous map $g: S^{3} \rightarrow \mathbb{R}$ there exists $x \in S^{3}$ such that $g(x)=g(\varphi(x))=g\left(\varphi^{2}(x)\right)$.

3. The particular case that $\varphi$ is an $\alpha$-contraction. In the proof of Theorem 1.1, since $A$ is a compact subset of $X$, it was possible to construct a compact subset $K$ of $A$ such that $\psi(K) \subset \varphi(K)$ (see (2.6)). In Lemma 3.4, we prove that even if $A$ is not compact, it is possible to ensure the existence of such a subset, provided $X$ is a metric space, $A$ is complete and $\varphi$ is an $\alpha$-contraction. Consider the following

Definition 3.1. Let $X$ be a normed linear space. For any bounded subset $A \subset X$, we define the measure $\alpha(A)$ of noncompactness of $A$ to be

$\alpha(A)=\inf \{k>0: A$ has a finite covering by sets of diameter $\leq k\}$.

Some important properties of $\alpha$ are given in the following proposition (for more details see, for example, [3] and [6]).

Proposition 3.2. Suppose $A, B$ are bounded subsets of $X$ and $k \in \mathbb{R}$. Then:

(1) $A \subset B$ implies $\alpha(A) \leq \alpha(B)$;

(2) $\alpha(A \cup B)=\max \{\alpha(A), \alpha(B)\}$;

(3) $\alpha(A+B) \leq \alpha(A)+\alpha(B)$;

(4) $\alpha(k A)=|k| \alpha(A)$;

(5) $\alpha(\operatorname{Co} A)=\alpha(A)$, where $\operatorname{Co} A$ denotes the convex hull of $A$;

(6) $\alpha(\bar{A})=\alpha(A)$, where $\bar{A}$ denotes the closure of $A$;

(7) $\alpha(A)=0$ if and only if $A$ is totally bounded.

Definition 3.3. Suppose $A$ is a subset of $X$ and $\varphi: A \rightarrow X$ is a continuous map. The map $\varphi$ is said to be an $\alpha$-contraction if there exists an $r, 0 \leq r<1$, such that $\alpha(\varphi(B)) \leq r \alpha(B)$ for any bounded subset $B$ of $A$. 
Lemma 3.4. Let $M$ be a metric space and $A$ a bounded and complete subset of $M$. Let $\psi, \varphi: M \rightarrow M$ be continuous maps such that $A$ is invariant under $\psi$ and $\varphi$ and $(\psi \circ \varphi)(a)=(\varphi \circ \psi)(a)$ for any $a \in A$. Then if $\varphi$ is an $\alpha$-contraction on $A$, there exists a compact subset $K \subset A$ such that $\psi(K) \subset \varphi(K)=K$.

Proof. Let $K$ be the intersection of subsets $K_{n}$ of $A$ inductively defined by $K_{1}=\overline{\varphi(A)}$ and $K_{n+1}=\overline{\varphi\left(K_{n}\right)}$. We will show that $\alpha(K)=0$, which implies by Proposition 3.2(7) that $K$ is totally bounded, and since $A$ is complete we conclude that $K$ is compact. In fact, for any $n \in \mathbb{N}$, since $\varphi$ is an $\alpha$-contraction, from Proposition 3.2(1) and (6) we have

$$
\begin{aligned}
\alpha\left(K_{n}\right) & =\alpha\left(\overline{\varphi\left(K_{n-1}\right)}\right)=\alpha\left(\varphi\left(K_{n-1}\right)\right) \leq r \alpha\left(K_{n-1}\right) \\
& \leq r^{2} \alpha\left(K_{n-2}\right) \leq \cdots \leq r^{n-1} \alpha\left(K_{1}\right) \leq r^{n} \alpha(A) .
\end{aligned}
$$

Since $K=\bigcap K_{n}$, we have $K \subset K_{n}$ for any $n \in \mathbb{N}$. It follows from Proposition 3.2(1) and from (3.1) that

$$
\alpha(K) \leq \alpha\left(K_{n}\right) \leq r^{n} \alpha(A), \quad \forall n \in \mathbb{N} .
$$

Since $0 \leq r<1$, we have $\lim _{n \rightarrow \infty} r^{n}=0$ and from (3.2) we conclude that $\alpha(K)=0$.

Now, we will show that $K=\varphi(K)$. It is easy to see that $\varphi(K) \subset K$. On the other hand, $K \subset \varphi\left(K_{n}\right)$ for any $n \in \mathbb{N}$. Let $x \in K$. Then $x=\varphi\left(x_{n}\right)$ for some $x_{n} \in K_{n}$. Let $S=\left\{x_{1}, x_{2}, \ldots\right\}$ and observe that $\alpha(S)=0$; thus $S$ is compact and so $\left(x_{n}\right)_{n \in \mathbb{N}}$ has a subsequence converging to some $y \in K$. Then $x=\varphi(y)$ and thus $K \subset \varphi(K)$. The condition $\psi(K) \subset K=\varphi(K)$ follows from the commutativity of the maps $\varphi$ and $\psi$ on $A$.

As a consequence of Lemma 3.4 we have the following version of Theorem 1.1 in the case that $\varphi$ is an $\alpha$-contraction.

Theorem 3.5. Let $M$ be a metric space and let $A$ be a bounded, complete, connected and locally pathwise connected subset of $M$. Let $\psi, \varphi$ : $M \rightarrow M$ be continuous maps such that $A$ is invariant under $\psi$ and $\varphi$. Suppose that

(i) $\psi_{*}-\varphi_{*}: i_{*}\left(H_{1}(A, \mathbb{Q})\right) \rightarrow i_{*}\left(H_{1}(A, \mathbb{Q})\right)$ is a surjective map;

(ii) $(\psi \circ \varphi)(x)=(\varphi \circ \psi)(x)$ for any $x \in A$.

Then for every continuous map $f: X \rightarrow \mathbb{R}^{2}$, either there exists a point $x \in X$ such that $f(\varphi(x))=f(\psi(x))$ or there exists a point $x \in X$ such that $f(\varphi \psi(x)) \in\left[f\left(\varphi^{2}(x)\right), f\left(\psi^{2}(x)\right)\right]$.

Proof. The arguments are similar to those used in the proof of Theorem 1.1: just observe that the existence of a compact subset $K$ of $A$ such that $\psi(K) \subset \varphi(K)$, as in (2.6), is ensured by Lemma 3.4. 
Acknowledgements. The authors are grateful to the referee for his valuable comments and suggestions.

\section{References}

[1] K. Borsuk, Drei Sätze über die n-dimensionale euklidische Sphäre, Fund. Math. 20 (1933), 177-190.

[2] D. G. Bourgin, On some separation and map theorems, Comment. Math. Helv. 29 (1955), 199-214.

[3] K. Deimling, Nonlinear Functional Analysis, Springer, Berlin, 1985.

[4] M. J. Greenberg, Lectures on Algebraic Topology, W. A. Benjamin, New York, 1967.

[5] S. T. Hu, Homotopy Theory, Pure Appl. Math. 8, Academic Press, New York, 1959.

[6] A. C. Ladeira, Continuity of fixed points, J. Math. Anal. Appl. 169 (1992), 350-358.

[7] E. Pannwitz, Eine freie Abbildung der n-dimensionalen Sphäre in die Ebene, Math. Nachr. 7 (1952), 183-185.

[8] P. L. Q. Pergher, D. de Mattos and E. L. dos Santos, The Borsuk-Ulam theorem for general spaces, Arch. Math. (Basel) 81 (2003), 96-102.

[9] C. T. Yang, On the theorems of Borsuk-Ulam, Kakutani-Yamabe-Yujobô and Dyson I, Ann. of Math. 60 (1954), 262-282.

Carlos Biasi and Denise de Mattos

Departamento de Matemática-ICMC

Universidade de São Paulo

Caixa Postal 668

13560-970, São Carlos SP, Brazil

E-mail: biasi@icmc.usp.br

deniseml@icmc.usp.br 